Musées, Patrimoine et Culture scientifiques et techniques

178 | 2018

juillet-août 2018

\title{
Vers la construction d'une société plus inclusive
}

\section{Caroline Buffet}

\section{OpenEdition \\ Journals}

Édition électronique

URL : http://journals.openedition.org/ocim/2664

DOI : $10.4000 /$ ocim.2664

ISSN : 2108-646X

Éditeur

OCIM

Édition imprimée

Date de publication : 1 juillet 2018

Pagination : 19-25

ISSN : 0994-1908

Référence électronique

Caroline Buffet, « Vers la construction d'une société plus inclusive », La Lettre de I'OC/M [En ligne], 178|

2018, mis en ligne le 01 juillet 2019, consulté le 10 décembre 2020. URL : http://

journals.openedition.org/ocim/2664; DOI : https://doi.org/10.4000/ocim.2664

Ce document a été généré automatiquement le 10 décembre 2020.

Tous droits réservés 


\section{Vers la construction d'une société plus inclusive}

\section{Caroline Buffet}

La reconnaissance tactile des œuvres d'art par les malvoyants complète la visite commentée du musée.

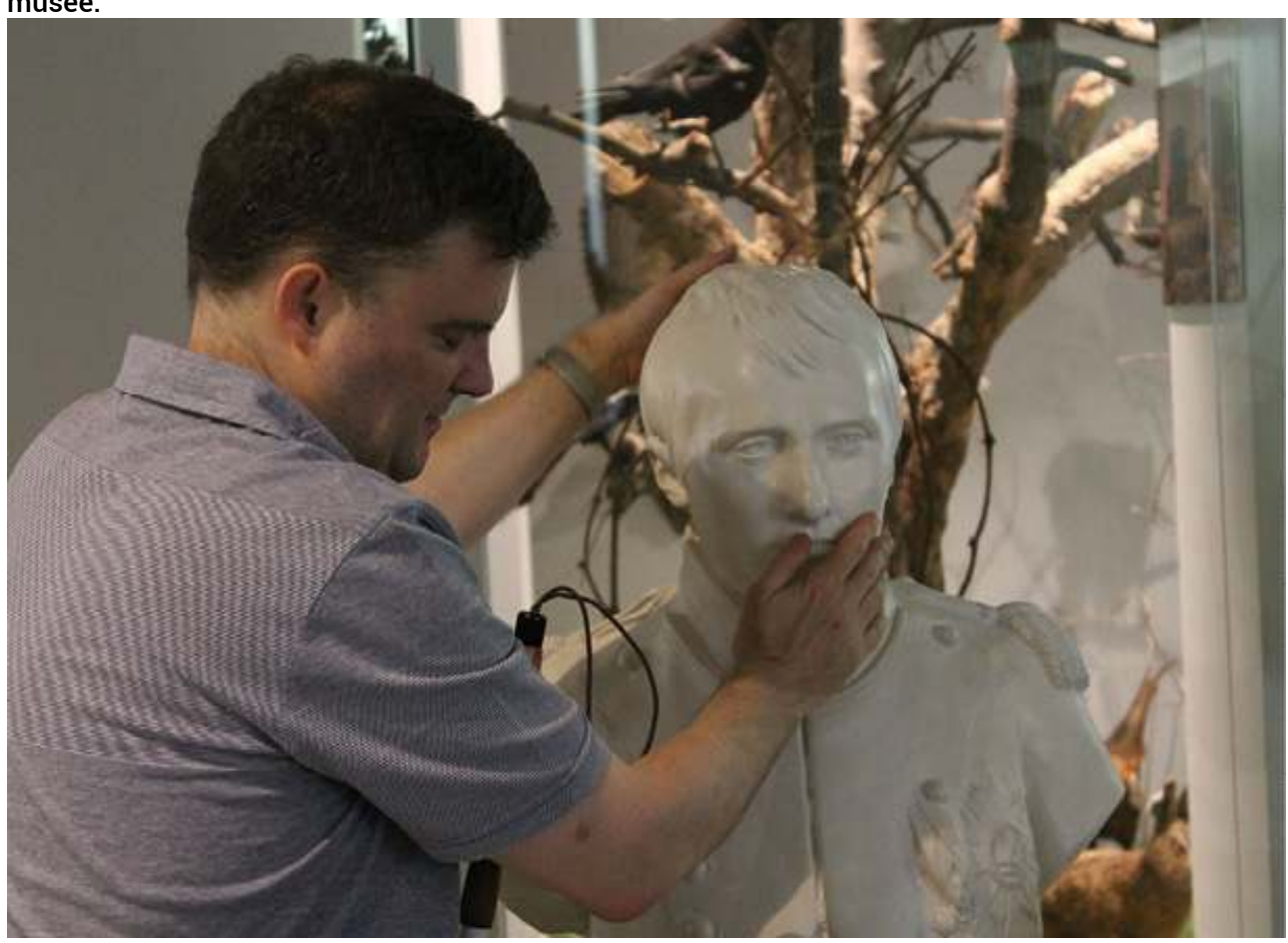

(c) Musée des Trois Pays Lörrach 


\section{Changer le regard sur le handicap : des aveugles guides conférenciers}

1 La Convention relative aux droits des personnes handicapées, ratifiée par la France le 18 février 2009, a pour objectif de "promouvoir, protéger et assurer" leurs droits : dignité, égalité devant la loi et jouissance des droits humains fondamentaux dont la participation active à la vie politique, économique, sociale et culturelle ${ }^{1}$. Quelques années plus tard, à l'issue de la Conférence nationale du handicap tenue en décembre 2014, trois objectifs principaux ont été fixés par le ministère des Solidarités et de la Santé : construire une société plus ouverte, concevoir des réponses et des prises en charge adaptées à la situation de chacun, simplifier la vie quotidienne.

Depuis, de nombreuses institutions culturelles dont les musées s'interrogent sur les implications qu'entrâne la construction d'une société plus inclusive pour leur politique d'accueil du public. Comment intégrer les visiteurs "empêchés"2 tout en garantissant leur droit de vivre dignement et de façon autonome avec leur handicap ? Notre article se penche sur la question des visiteurs déficients visuels. Un défi pour les musées quand il s'agit de faire découvrir des pièces purement visuelles à des personnes qui ne les voient pas tout en respectant les conditions requises de sécurité, de climat et de lumière.

3 Le défi est cependant passionnant : concevoir des animations variées accessibles à tous les visiteurs, quels que soient leur âge, origine, niveau de connaissances et bien entendu aptitudes physiques ou mentales. L'attente des personnes empêchées est avant tout celle de l'intégration : se sentir bienvenues et considérées comme tout un chacun. Nous avons constaté au musée de Lörrach que nous ne pouvions pas satisfaire ce souhait avec des animations spécifiques - et donc exclusives - tenant compte des divers types de handicap. Quoi de plus frustrant pour une personne aveugle aspirant à un programme inclusif que de se retrouver dans une pièce à part du musée réservée à l'appréhension tactile et au milieu d'autres aveugles $?^{3}$ Avec les meilleures intentions, les responsables de ce genre de manifestations s'enfoncent malheureusement un peu plus loin dans la direction de l'exclusion.

4 La question de l'inclusion nous incite alors à réviser nos traditionnels modes de médiation et à en inventer d'autres à l'adresse de tous. Il faut reconsidérer l'expérience de visite dans sa globalité, tenir compte des effets du langage corporel et de l'acoustique, dépoussiérer un jargon routinier et y intégrer des expressions suscitant l'imaginaire, détrôner la suprématie de la vision et exploiter l'approche sensorielle par le toucher, l'ouïe, l'odorat et le goût. C'est ce que propose par exemple, le service de médiation du musée de l'Homme avec un parcours sensoriel basé sur une vingtaine de supports originaux répartis dans la Galerie de l'Homme. Il est composé d'objets à toucher, de commentaires sonores et de plateaux tactiles qui à eux tous permettent de découvrir les thématiques abordées dans le musée et de découvrir l'histoire de l'humanité4. Il existe maints autres exemples d'application de l'approche multi sensorielle dans les musées.

\section{La Fédération des Aveugles et Amblyopes de France}

Elle recense environ 1,7 million de personnes atteintes d'un trouble de la vision. Parmi elles, 207000 sont aveugles (pas de perception de la lumière) et malvoyantes profondes (vision résiduelle limitée à la distinction de silhouettes). Les autres 
souffrent d'une incapacité visuelle sévère : en vision de loin, ils ne peuvent pas distinguer un visage à 4 mètres et en vision de près, la lecture est impossible. Étant donné le vieillissement de la population, ces taux ne feront que s'accroître au cours des prochaines décennies.

\section{Le projet "Conte invisible"}

\section{Médiateurs et visiteurs de musée - Et si on inversait les rôles ?}

Lors d'une journée rencontre sur le thème "Tourisme et handicap" au musée Électropolis de Mulhouse en automne 2015, nous avons rencontré Nicolas Caraty. Né malvoyant, il a perdu la vue à 14 ans ce qui ne l'a pas empêché de se lancer des défis toujours plus audacieux et d'exercer des métiers très divers : tour à tour membre de l'équipe de France de football aveugle, téléconseiller, standardiste, accordeur de piano, il a aujourd'hui un peu plus de 45 ans et occupe le poste de guide médiateur et responsable du service accessibilité au musée d'Aquitaine de Bordeaux. Il est probablement le seul non-voyant en France à exercer ce métier. Son aisance est fascinante et montre combien il a réussi à surmonter et même à valoriser son handicap. Cette rencontre m'a fortement impressionnée et m'a donné l'idée de faire participer activement des personnes nonvoyantes dans mon travail de médiation au musée des Trois Pays. On peut aussi citer l'exemple d'Alexis Dussaix, un jeune homme sourd qui a étudié l'histoire de l'art à l'École du Louvre pour devenir guide-conférencier en langue des signes 5 .

\section{Ébauche et mise en place du projet}

6 À peine formulée, l'idée de donner la parole à des médiateurs aveugles a soulevé à la fois enthousiasme et scepticisme dans notre entourage aussi bien privé que professionnel et il s'est vite avéré que la réalisation d'un tel enjeu n'aboutirait pas sans difficulté. Quiconque - qu'il soit voyant ou non voyant - ne possède pas le remarquable talent de médiateur de Nicolas Caraty. Ses visites guidées sont le fruit d'un travail de longue haleine : pendant un an, il a pu s'approprier les objets préhistoriques exposés dans les vitrines du musée d'Aquitaine et les explorer autant que possible par le toucher. Parallèlement, il a pu se procurer et étudier toute une documentation numérisée pour connaître l'histoire de ces pièces archéologiques et construire le plan de son parcours guidé. Il se déplace aujourd'hui tout à son aise dans les salles du musée. Captivés, les visiteurs l'écoutent narrer l'histoire de ces objets qu'il n'a jamais vus'.

\section{D'autres types de scénarios envisageables}

On pourrait par exemple imaginer la description d'un groupe d'œuvres plastiques. L'expérience a été faite au musée d'Orsay en février 2015. Nadine Dutier, ergothérapeute auprès de la Fédération des aveugles de France, a proposé au musée d'Orsay à Paris de raconter l'histoire d'une œuvre impressionniste sur une musique de Debussy pour donner un élan à des explications monocordes : "Le travail autour du conférencier est fondamental", explique-t-elle, "il doit exprimer ses émotions, dire pourquoi il aime ou non telle ou telle cuvre. La projection des visiteurs est alors immédiate et l'écoute 
active". Avec le même objectif, certains essaient de théâtraliser leurs explications au maximum pour plonger les visiteurs dans l'univers du tableau.

\section{Premier essai et bilan}

7 Dans un premier temps, nous avons contacté Dirk Furtwängler, un fidèle visiteur du musée, né malvoyant et devenu aveugle à 13 ans, et lui avons proposé d'élaborer ensemble un projet de médiation. Nous avions rassemblé quelques pièces de la collection du musée pour lui permettre d'expérimenter la découverte haptique (ou tactile) : un costume féminin alsacien traditionnel en textile, une cuirasse médiévale en métal, une sculpture en grès représentant sainte Catherine, une statue en bois du célèbre poète local Johann Peter Hebel et un buste en plâtre de Napoléon Bonaparte. Toutes ces figures avaient en commun leur taille, presque humaine, et pouvaient facilement être explorées tactilement. Nous pensions naïvement qu'en les rapprochant les unes des autres, elles allaient "communiquer entre elles" et qu'à partir de sa perception haptique, Dirk allait composer un dialogue original. L'idée était avant tout de faire ressortir ce qu'il percevait en tant que non-voyant, que nous ignorions en tant que voyante.

Il s'agissait d'exploiter les déductions de la perception tactile et de les croiser avec celles de la vision. Nous nous demandions par exemple si, en inspectant le visage de Napoléon par le toucher, Dirk allait identifier certains traits de caractère du célèbre empereur, voire même détecter un aspect jusque-là laissé de côté ? Nous lui avons suggéré de prendre le temps de découvrir ces objets, de les toucher sans retenue pour se les "approprier", de laisser libre cours à son imagination, de commenter ce qu'il ressentait et d'élaborer une sorte de scénario. À la fin de cette première séance, il a exprimé sa réticence car l'exercice proposé dépassait largement ses compétences. Nous avons alors pris conscience de l'ampleur du défi : parler seul et librement d'objets qu'on ne voit pas à un public voyant mais invisible et dont on ne connaît ni les attentes ni les exigences est un véritable exploit qui suppose de longues heures d'entraînement. L'année de préparation dont Nicolas Caraty a disposé pour préparer ses visites est parfaitement calculée et légitime - un temps de travail qui ne peut pas se réduire à quelques heures. Pour contourner cette difficulté, Dirk a souhaité inviter une seconde personne, Ulrike Seis, elle aussi aveugle, à participer au "jeu" : pour affronter l'inconnu, on est mieux armé à deux que seul. Par ailleurs, pour un médiateur débutant, il est évidemment plus facile de parler d'un sujet familier. Les objets sélectionnés pour la première séance étaient certes intéressants d'un point de vue haptique, mais appartenaient à un domaine trop étranger pour Dirk. Le costume de l'Alsacienne lui était par exemple totalement inconnu. N'arrivant pas à l'identifier, il s'est senti mal à l'aise, un peu honteux de son ignorance et a même craint de vexer quelqu'un de l'assistance qui aurait pu d'après lui "s'offusquer qu'on ne reconnaisse pas le costume traditionnel local." Cette préoccupation témoigne de l'extrême susceptibilité des aveugles et de leur isolement. À la recherche d'un thème moins aventureux, l'idée du conte de fée a été retenue et nous avons choisi celui des frères Grimm Hänsel et Gretel. Mise à part l'association entre le pain d'épice et la période de Noël dans laquelle nous nous trouvions à l'époque, aucune raison particulière n'a fondé ce choix. Le fait de s'adresser à des enfants - qui selon Dirk et Ulrike sont moins exigeants que des adultes - les rassurait. Une fois ces nouvelles conditions arrêtées - 
narrer librement un conte célèbre à deux et à un public d'enfants - le montage du projet a pu se poursuivre.

Au musée des Trois Pays Lörrach, Le Conte invisible permet à des aveugles guides conférenciers d'être des médiateurs auprès d'enfants placés dans la condition de malvoyants.

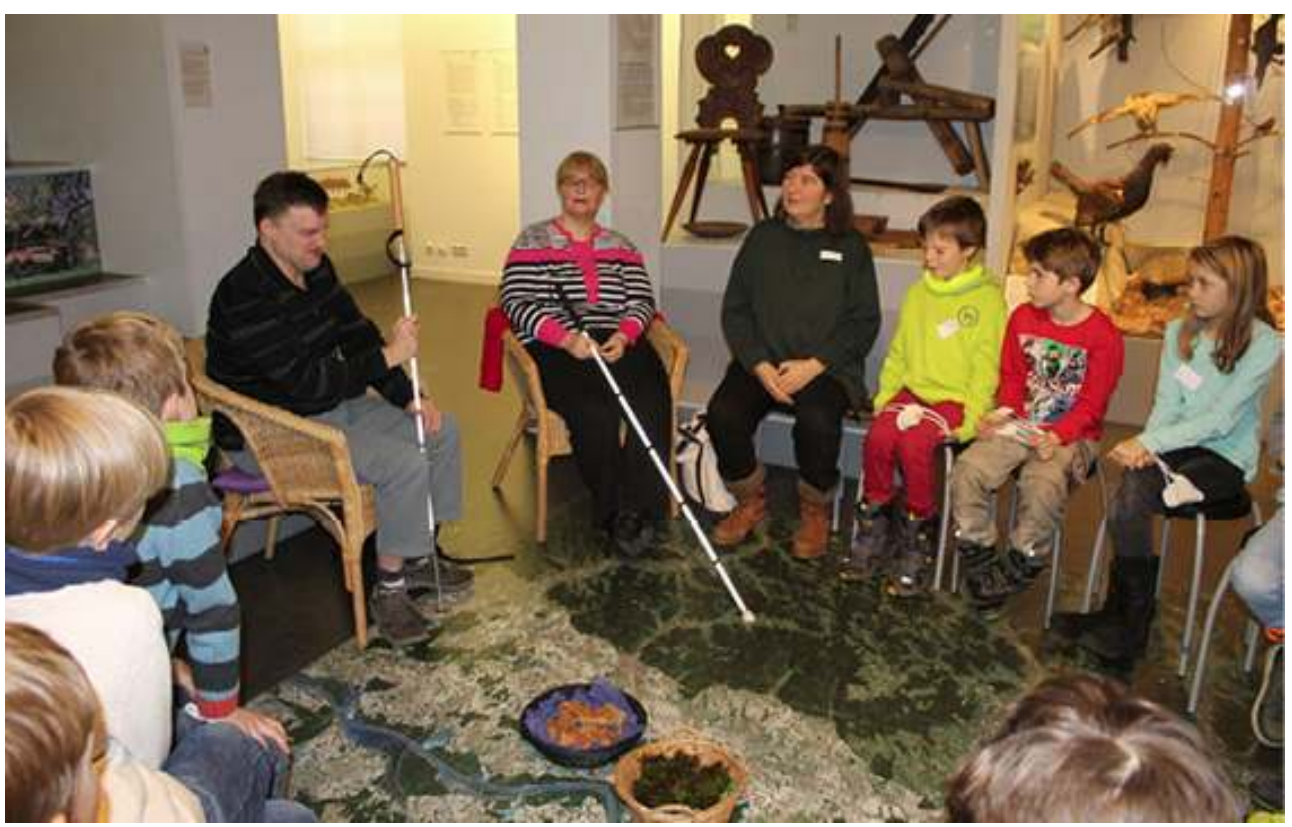

(c) Musée des Trois Pays Lörrach

Au musée des Trois Pays Lörrach, Le Conte invisible permet à des aveugles guides conférenciers d'être des médiateurs auprès d'enfants placés dans la condition de malvoyants.

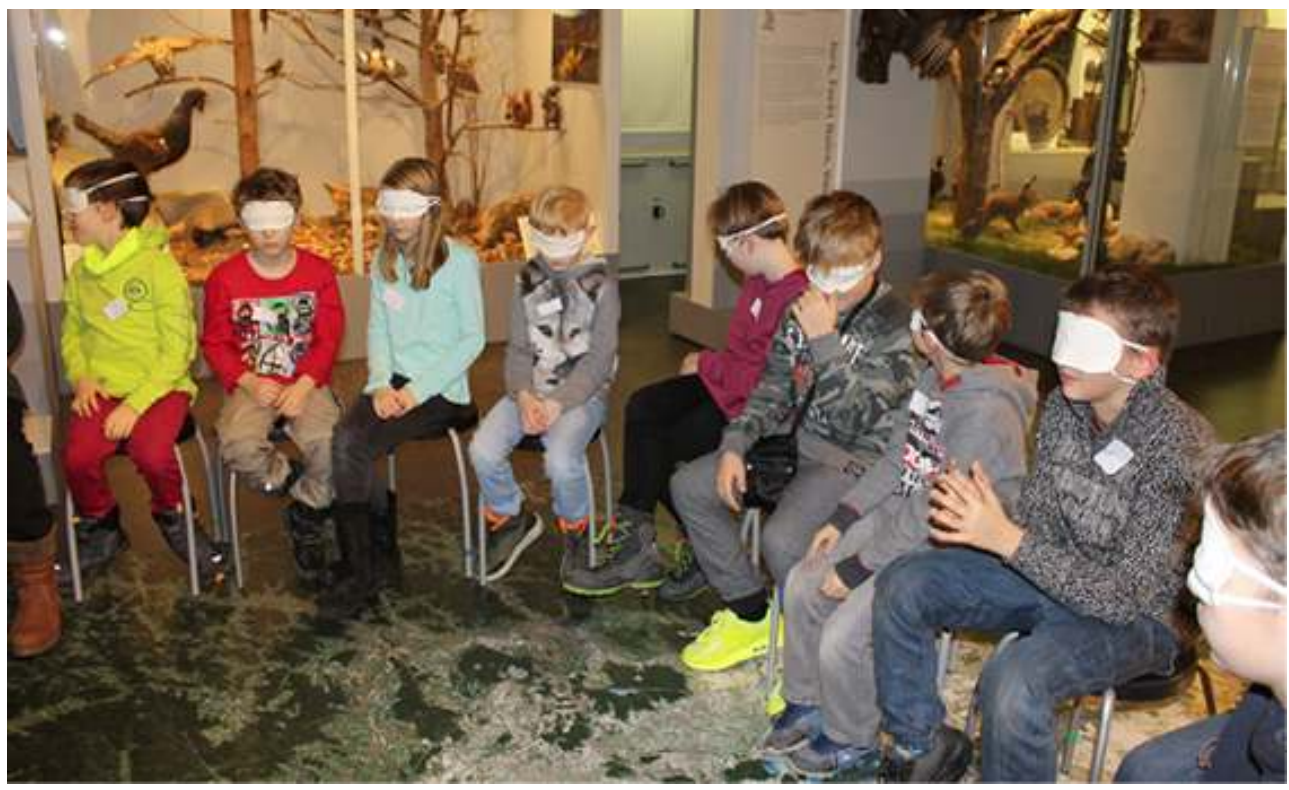

(C) Musée des Trois Pays Lörrach 


\section{Seconde expérience}

\section{Un public confiant et rassurant}

Pour passer à la seconde étape du projet, il fallait trouver le cadre dans lequel cette manifestation allait pouvoir se dérouler. Une fois par mois, le service médiation du musée des Trois Pays anime une séance "jeunes détectives" pour les enfants de 7 à 12 ans. Le thème varie à chaque fois et se démarque des habituels critères pédagogiques. Ce programme est réservé aux jeunes visiteurs et leurs parents ne sont pas censés y assister. Il comporte deux objectifs essentiels : d'une part faire découvrir le musée et les facettes insoupçonnées de sa collection aux enfants et d'autre part leur faire rencontrer d'autres enfants du même âge qui fréquentent d'autres établissements scolaires et habitent dans d'autres quartiers. Cette animation mensuelle du samedi après-midi accueille en général une quinzaine de participants. Elle n'a rien de scolaire et constitue donc un cadre idéal pour expérimenter des projets de médiation un peu hors du commun - comme celui du conte narré par des aveugles. Dans l'invitation adressée quelques jours à l'avance aux parents, il n'avait pas été mentionné que les deux conteurs étaient aveugles. Le titre mystérieux "conte invisible" devait suffire pour attiser la curiosité. Treize enfants âgés de 6 à 10 ans ont répondu à l'appel.

Les enfants reconnaissent les objets avec l'un des 4 sens : haptique, olfactif, gustatif, auditif.

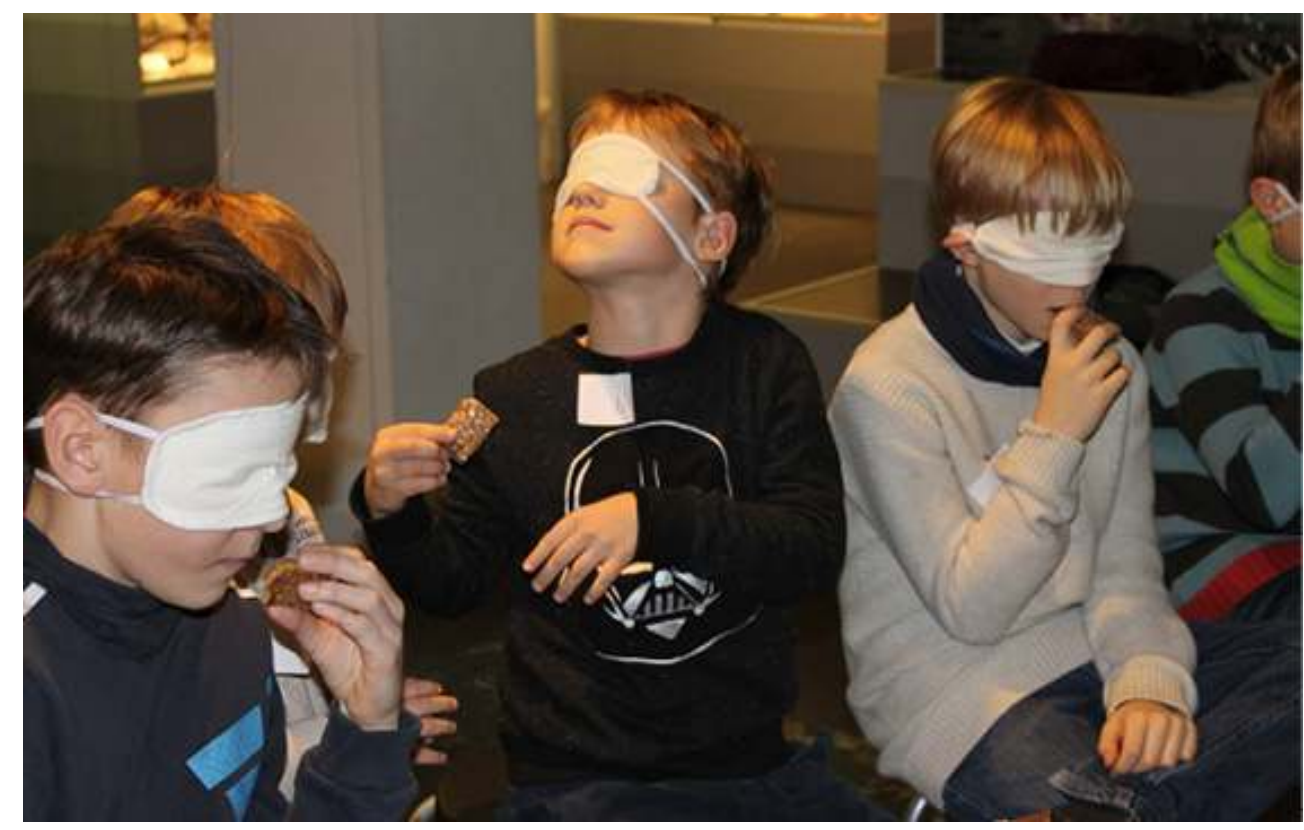

(c) Musée des Trois Pays Lörrach 
Passage des objets à identifier durant la narration.

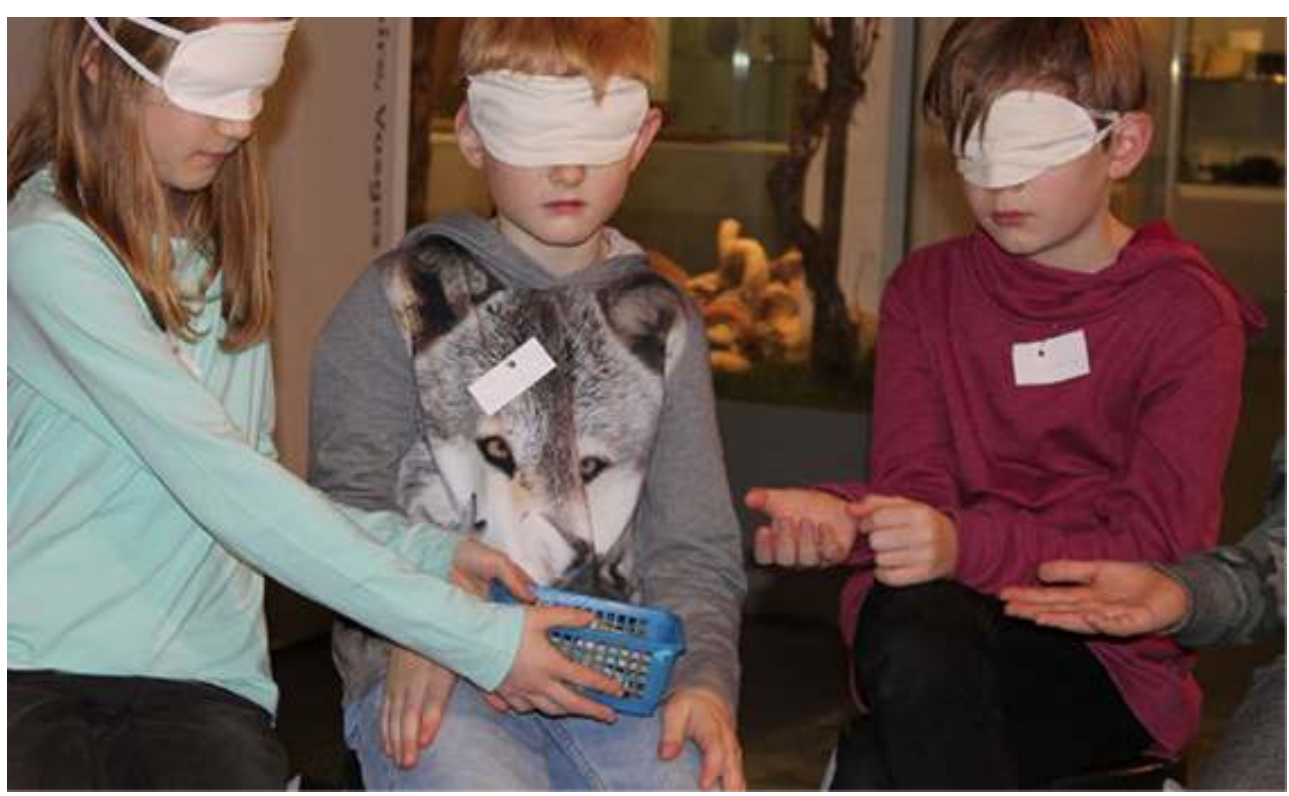

(c) Musée des Trois Pays Lörrach

\section{Une scénographie à suspens}

Une fois installés autour des deux conteurs, les enfants se sont présentés en indiquant leur prénom et leur âge. Nous avions demandé à l'une de nos collègues médiatrices de nous assister. Pour leur permettre d'entrer dans le monde aveugle, nous leur avons proposé de se bander les yeux. Tous ont accepté - un peu intrigués et séduits en même temps par ce caractère expérimental. La narration a pu ensuite commencer : Dirk et Ulrike prenaient la parole à tour de rôle et spontanément. Comme nous en avions préalablement convenu, ils faisaient une pause de temps à autre qui laissait le temps aux jeunes auditeurs de découvrir divers objets qui leur passaient entre les mains : ils ne les voyaient pas et devaient les identifier par l'un ou l'autre des autres sens, le toucher, l'ouie, l'odorat et le goût. "Les parents complètement démunis d'Hänsel et Gretel (quignon de pain sec) décident de les perdre dans la forêt. Mais Hansel est astucieux (petits cailloux) et retrouve le chemin de la maison. Les parents renouvellent l'opération un peu plus tard. Hänsel ayant trouvé la porte close imagine une autre solution (miettes de pain) qui cependant échoue (petits oiseaux naturalisés). Les deux enfants errent dans la forêt (odeur de mousse fraîche, hululement de la chouette, bruit de feuilles sèches et craquement de branches) et arrivent finalement à une cabane insolite (pain d'épice, cannelle) habitée par une femme qui s'avère être une méchante sorcière. Elle fait durement travailler Gretel (écoulement de l'eau puisée) et enferme Hänsel dans une cage pour le faire grossir puis le dévorer (os). Gretel parvient à enfermer la sorcière dans le four brûlant et à libérer son frère. Avant de repartir, ils inspectent la cabane et $y$ découvrent un trésor (pierres précieuses, pièces de monnaie). Ils rencontrent un grand oiseau (canard naturalisé) qui leur fait traverser la rivière et les aide à retrouver le chemin de leur maison". 


\section{La cécité à bâtons rompus}

11 La narration a duré 40 minutes au cours desquelles les enfants ont dû utiliser les quatre sens tactile, auditif, olfactif et gustatif pour appréhender des objets qui illustraient l'une ou l'autre facette de l'histoire. Les jeunes auditeurs sont restés concentrés durant tout le temps de la narration et n'ont manifesté ni ennui ni impatience. Une fois le conte fini, ils ont pu enlever leur bandeau et une discussion animée autour de ce qu'ils venaient de vivre s'est aussitôt engagée. À la question sur la différence entre l'écoute d'une histoire les yeux fermés avec celle les yeux ouverts, ils ont unanimement répondu qu'ils étaient mieux concentrés avec les yeux bandés et que les objets qu'ils avaient ressentis avaient fait naître des images nouvelles. Tous connaissaient déjà plus ou moins le conte d'Hänsel et Gretel avant la séance, mais l'ont redécouvert sous une autre perspective, principalement celle du sens haptique. Les enfants ont ensuite pu poser librement et sans aucune gêne leurs questions aux deux conteurs : comment vous orientez-vous dans la ville? Vous arrive-t-il de vous perdre ? Comment faites-vous vos courses? Avez-vous un chien? Regardez-vous la télévision? Comment savez-vous à quel moment vous pouvez traverser la rue ? Quel est votre métier et où travaillez-vous ? Dirk et Ulrike ont répondu ouvertement et sans retenue. Ils ont aussi expliqué le principe de l'écriture Braille et fait circuler divers documents que les enfants ont pu explorer tactilement. La discussion a duré plus de 40 minutes, mais d'autres questions étaient encore ouvertes quand leurs parents sont venus les chercher.

Entraînement au système de lecture Louis Braille

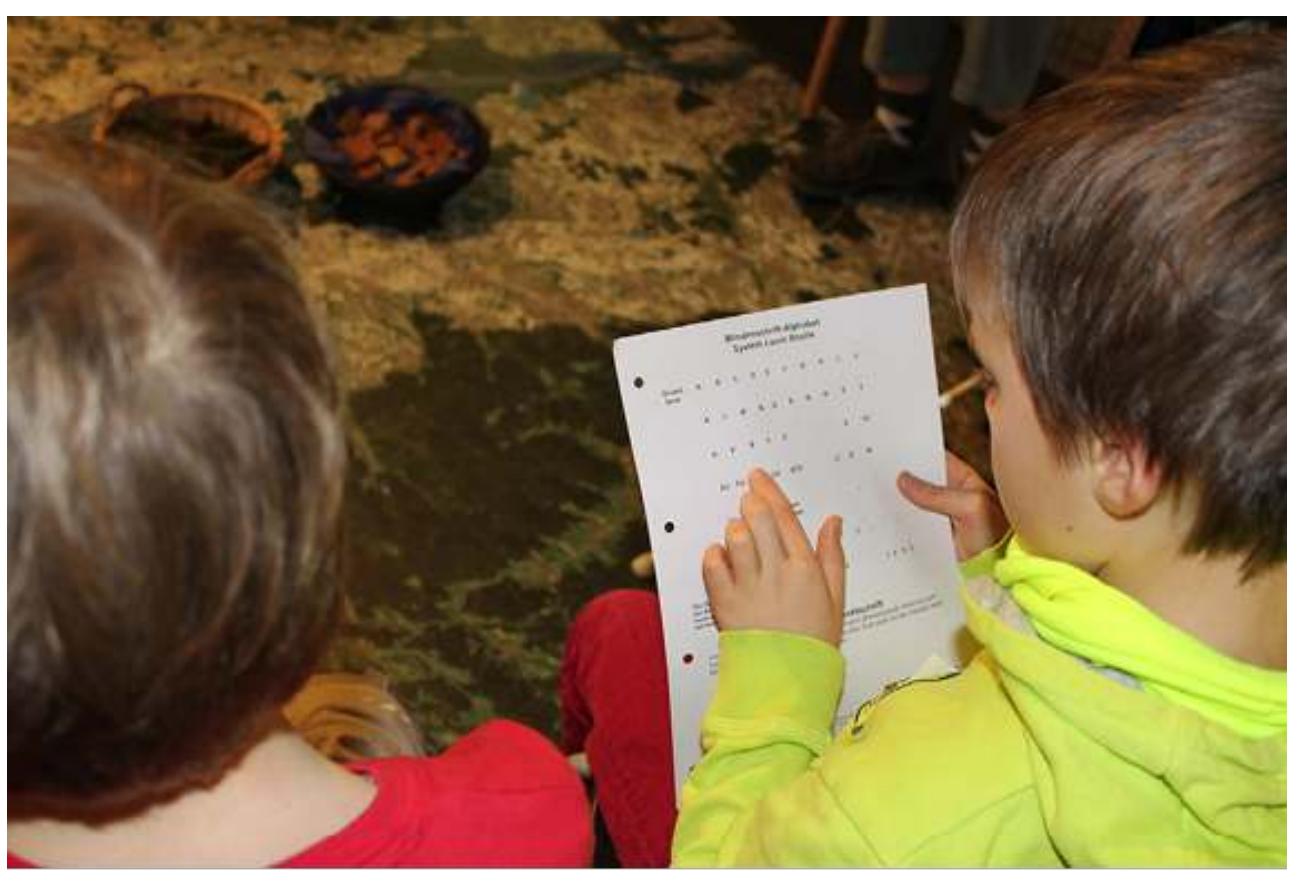

(c) Musée des Trois Pays Lörrach

"Prière de ne pas toucher" et l'approche tactile des objets exposés

Pour des raisons de conservation préventive que nous connaissons tous, une distance mesurée doit être maintenue entre l'œuvre et le visiteur - 
étymologiquement celui qui "va voir". Celle-ci peut être physique par le moyen de barrières, de vitres ou de vitrines ou humaine avec un personnel de surveillance active et constante des salles ouvertes aux visiteurs. L'interdiction de toucher découle d'une prise de conscience générale de la fragilité des pièces d'un patrimoine souvent unique : les objets exceptionnels doivent être conservés dans les meilleures conditions possibles pour pouvoir être transmis aux générations futures. Parmi les facteurs de dégradation, on trouve la lumière, la poussière, les variations brusques de température et d'humidité, mais aussi et surtout les manipulations humaines. Un simple doigt sur un objet peut déposer des poussières, de la sueur, des traces de savon ou de crème, voire des microorganismes (moisissures, bactéries...) : il abîme donc irrémédiablement ce dernier, surtout si ce geste est répété des milliers de fois ${ }^{7}$.

Par ailleurs, il n'est plus nécessaire de démontrer que le toucher est essentiel pour voir et comprendre l'univers qui nous entoure. Ce phénomène est valable pour tous les visiteurs, et pas seulement pour les personnes aveugles. Il fonde de nombreuses offres de médiation culturelle des musées qui cherchent à s'adresser au plus grand nombre : toucher, expérimenter, interagir contribuent à la compréhension et à l'appropriation des objets.

Comment contourner alors cette incompatibilité totale entre l'impératif de préserver les objets et celui de les rendre accessibles en permettant au public mal ou non-voyant de les toucher? Un aveugle muni de gants est doublement amputé de ses facultés : il ne peut ni voir ni toucher. Actuellement, les progrès technologiques et la démocratisation de l'impression 3D ouvrent de réelles perspectives dans le domaine du handicap visuel. Des plans reliefs imprimés, des plaques ou encore des téléphones en braille et bien d'autres projets peuvent matérialiser un monde inaccessible aux non-voyants. Les expériences de divers services de médiation méritent d'être ici citées.

Celui du musée du Louvre a organisé durant plusieurs années des visites de la Galerie Tactile à l'intention des groupes de visiteurs aveugles et malvoyants. Guidés par un conférencier spécialement formé, il leur était possible de toucher certaines œuvres sculpturales en pierre très dure et supportant donc le contact. Ces visites avaient lieu le mardi, jour de fermeture du musée, à l'abri du regard du grand public qui n'aurait pas compris la raison d'une telle entorse à la règle.

Celui du LaM-Lille Métropole, musée d'art moderne à Villeneuve-d'Ascq, accueille des enfants et adultes mal et non-voyants et leur propose différentes activités pour appréhender le musée "avec le bout des doigts". Elles sont animées par des médiateurs formés "à trouver les mots justes pour rendre visible la parole". Au programme se trouvent des parcours tactiles autour de sculptures originales d'artistes majeurs des collections. Elles peuvent être touchées selon certaines règles définies avec les conservateurs (port de gants dans certains cas, passage individuel...). Le service propose aussi des interprétations de tableaux en relief grâce à la technique du thermo-gonflé qui permet d'exécuter des images tactiles avec lesquelles le visiteur peut construire progressivement une représentation mentale d'œuvres peintes, dessinées ou photographiées. 
Celui du muséum d'Histoire naturelle de Bâle en Suisse a mis au point le programme permanent "Prière de toucher !" à l'intention de tous les visiteurs et plus particulièrement des non-voyants. Il s'agit de bornes accessibles sur lesquelles sont présentés des spécimens de taxidermie pouvant être touchés. Ils sont accompagnés de textes écrits en braille et en gros caractères. Il est toujours édifiant de voir le grand intérêt qu'y portent les visiteurs voyants ${ }^{9}$.

\section{Bilan du projet}

12 L'animation s'est déroulée le 16 décembre 2017. Lors de la réflexion qui a suivi, les deux conteurs ont tout de suite exprimé leur plaisir et signalé leur disponibilité pour renouveler l'expérience. Celle-ci est programmée pour décembre 2018 avec un nouveau "conte invisible" et va ainsi s'inscrire au programme permanent du service de médiation. Deux améliorations essentielles nous apparaissent nécessaires pour maintenir le dynamisme de l'animation tout au long de son déroulement. D'une part, les conteurs devront s'entraîner pour assurer le suspens de la narration : parler plus fort et adapter leur expression à leur public spécifique, en l'occurrence celui des enfants. D'autre part, nous avons constaté que, quand au bout d'une à deux minutes ils n'ont plus rien ni à écouter ni à toucher, les enfants se déconcentrent facilement. Cela nécessite l'assistance d'une ou deux personnes voyantes qui rythment le passage des objets et "meublent" les temps de passivité. Par ailleurs, certains des objets, notamment les pièces de la collection du musée autres que les accessoires, doivent être manipulés avec soin. Nous avions par exemple sorti un moineau naturalisé pour illustrer le moment où Hänsel et Gretel ne retrouvent plus les miettes de pain que les oiseaux avaient mangées. Le petit spécimen d'ornithologie - même si parce que sans grande valeur il peut être mis à la disposition des ateliers pédagogiques - requiert d'être touché et caressé avec délicatesse. Chaque enfant devrait alors disposer d'au moins 10 à 20 secondes pour identifier et comprendre ce qui lui passe entre les mains, un temps dont il faut tenir compte pour aménager le dynamisme de l'animation. Les deux conteurs ont été rémunérés pour leur intervention. Ils ont tous deux accepté les améliorations que nous avons suggérées notamment celle de s'entraîner à la narration.

13 L'expérience est originale et pourrait être conduite dans tous les musées quels que soient leurs profils, dans le cadre des expositions permanentes ou temporaires. Le musée des Trois Pays qui raconte l'histoire culturelle et politique de la région transfrontalière entre la Suisse, l'Allemagne et la France n'est pas plus approprié qu'un autre pour mener ce genre de projet. Les collections de la plupart des musées qu'ils soient d'histoire naturelle, ethnologique, archéologique, technique ou de l'art regorgent d'objets intéressants du point de vue haptique qui gagneraient à être sortis des réserves. Le thème des contes de fées est lui aussi d'une grande richesse et peut nourrir des discussions passionnantes aussi bien avec les enfants qu'avec les adolescents et les adultes. Ils permettent d'aborder des aspects éthiques, philosophiques, historiques et bien d'autres encore comme le vécu personnel. D'autres types de scénarios seraient envisageables comme par exemple une anecdote de l'histoire locale. Il existe à Lörrach la légende d'une cruelle sorcière qui préparait des potions à base de plantes venimeuses. Elle se prêterait parfaitement à une visite pluri sensorielle. Après la narration, il peut s'ouvrir une discussion intense au cours de laquelle conteurs et auditeurs, voyants ou non-voyants, racontent leur expérience et 
échangent à bâtons rompus. Par là-même se pose un autre regard sur le handicap. Les mots de Luis Jorge Borges, écrivain poète argentin aveugle, prennent alors toute leur signification : "Le monde de l'aveugle n'est pas la nuit que les voyants imaginent". Par ailleurs, la tradition veut qu'Homère, conteur poète grec du VIIIe siècle avant notre ère et auteur des deux célèbres épopées qui sont au fondement de la littérature occidentale, ait été aveugle. On ne peut qu'être frappé par la fréquence de l'association entre cécité et poésie.

\section{Pour la construction d'une société plus inclusive}

Une coopération régulière et soutenue avec les publics empêchés pour la conception des visites guidées et ateliers pédagogiques ne peut qu'en assurer une meilleure qualité. Elle nous conduit à remettre en question nos méthodes traditionnelles de médiation. Cette transformation ne doit pas faire peur : les attentes et compétences des visiteurs évoluent avec le temps et nous devons nécessairement nous adapter, actualiser nos schémas classiques, tenir compte des nouveaux comportements sociaux et des modernisations technologiques. N'hésitons pas à être créatifs et audacieux, osons la fantaisie et l'expérimentation dans le domaine des relations humaines, de l'expression et de la réception. Nous rassurons ici les directeurs de musées : cette révision des techniques de médiation n'est pas nécessairement coûteuse ! Elle se mesure avant tout qualitativement dans le sens où elle répond à la mission sociale du musée en tant que fédérateur de solidarités et contribue à l'attachement durable et fidèle d'un public plus large et plus diversifié. Qu'ils soient empêchés ou non, tous les visiteurs et acteurs du musée peuvent en profiter.

Fourès, A., Grisot, D. et Lochot, S. (dir.) Le rôle social du musée : agir ensemble et créer des solidarités. Dijon : Ocim, 2011, 196 p.

Stocker Steinke, S. et Staub, J. Praxishandbuch für ein Museum ohne Barriere. Baden : Hier und Jetzt, Verlag für Kultur und Geschichte GmbH, 2017, 160 p.

\section{NOTES}

1. Elle a été adoptée par l'Assemblée générale des Nations Unies le 13 décembre 2006 et est entrée en vigueur le 3 mai 2008.

2. On entend par public "empêché", les visiteurs en situation de handicap et donc éloignés d'un accès à la culture que ce soit pour des raisons physiques, psychologiques ou sociologiques.

3. Leysen Joyce, "Blindness and 'The object of art-in-between'. Towards cosmopolitical encounters in museological settings" 12/06/2017, téléchargeable à l'adresse www.public disabilityhistory.org/2017/12/blindness-and-object-of-art-in-between.html\#more.

4. www.museedelhomme.fr/fr/visitez/accessibilite/visiteurs-aveugles-malvoyants.

5. Interview de Bernard Hasquenoph publié le 30/03/2015 téléchargeable à l'adresse http:// louvrepourtous.fr/Alexis-Dussaix-l-histoire-de-l-art,784.html.

6. https://france3-regions.francetvinfo.fr/nouvelle-aquitaine/gironde/nicolas-caraty-nonvoyant-et-guide-au-musee-d-aquitaine-593138.html. 
7. Intervention de Françoise Feger et Lilia Jatlaoui (Service Éducation, musée du Louvre) en introduction à la journée Partages 2013 : "Du Ne pas Toucher à la médiation multisensorielle", décembre 2013 téléchargeable à l'adresse www.louvre.fr/sites/default/files/medias/ medias_fichiers/fichiers/pdf/louvre-introduction-partages-2013-pas.pdf.

8. www.musee-lam.fr/archives/11450

9. www.nmbs.ch/info_angebote/menschen-mit-besonderen-beduerfnissen.html

\section{RÉSUMÉS}

Dans les institutions muséales, la question de l'inclusion incite les responsables de la médiation à réviser leurs méthodes traditionnelles et à en inventer d'autres à l'adresse notamment des visiteurs non-voyants : l'expérience du musée des Trois Pays Lörrach, relatée ici sous forme de témoignage, utilise la dimension multi-sensorielle et principalement celle du toucher.

\section{INDEX}

Mots-clés : Société, inclusif, expérience

\section{AUTEUR}

\section{CAROLINE BUFFET}

Responsable de la médiation culturelle au musée des Trois Pays Lörrach en Allemagne.

c.buffet@loerrach.de 